\title{
COMPUTATIONAL TOPOLOGY TECHNIQUES HELP TO SOLVE A LONG-LASTING FORENSIC DILEMMA: ALDO MORO'S DEATH
}

James F. Peters

Department of Electrical and Computer Engineering, University of Manitoba

75A Chancellor's Circle, Winnipeg, MB R3T 5V6, Canada

james.peters3@umanitoba.ca

\author{
Arturo Tozzi (Corresponding Author) \\ Center for Nonlinear Science, Department of Physics, University of North Texas, Denton, Texas 76203, USA \\ 1155 Union Circle, \#311427, Denton, TX 76203-5017 USA \\ Computational Intelligence Laboratory, University of Manitoba, Winnipeg, Canada \\ Winnipeg R3T 5V6 Manitoba \\ tozziarturo@libero.it
}

\begin{abstract}
Here we show how a recently-introduced method from algebraic topology, namely proximal planar vortex 1-cycles, might be helpful in detecting hidden features of the shapes and holes in images, therefore contributing to the solution of both cold and fresh forensic cases. In particular, we test the efficacy of this technique by assessing one of the most puzzling cases of recent history, i.e., Aldo Moro's death. Terrorists of the Red Brigades claimed that they killed Moro when he was placed inside the trunk of a car,shooting him with a barrage of bullets. We demonstrate, based on the analysis of the photographs taken during the autoptic procedure, that the terrorist's account does not hold true. Our results, showing different series of shots, point towards a three-step execution, with the first phasestaking place outside the car. In conclusion, the novel forensic analysis method introduced in this paper permits the evaluation of a collection of vortex cycles/nerves equipped with a connectedness proximity, which makes it possible to assess unexpected spatial clusters in photographs.
\end{abstract}

KEYWORDS: tessellation; forensic picture; bullets; homology; proximity

\section{INTRODUCTION}

The kidnapping of Aldo Moro, the promoter of the alliance between centrists and communists in the Cold War Era, was a defining moment in Italian political history (Drake 1995; Martinelli and Padellaro, 1979; Montanelli and Cervi,1991). At 9 o' clock of 16 March 1978, Moro, former prime minister and then President of the relative majority party Christian Democracy, was kidnappedin Rome by left-terrorists of the of Red Brigades (Sciascia 1994; Gotor 2018). After 55 days of imprisonment, Moro's body was foundin the trunk of a red Renault 4 in Via Caetani (Zavoli1992). The President was murdered in the morning of 9 May 1978 (Biscione 1993; Grassi 2008). According to the official version, confirmed by the same brigatistsabout ten years after the event, the killers placed him into a wicker basket and brought him to the parking garage in via Montalcini (Satta 2006). They inserted him into the trunk of a red Renault, then they shot him with two weapons, a $9 \mathrm{~mm}$ Walther PPK and a $7.65 \mathrm{~mm}$ Škorpionvz. 61. The car with his corpse was parked in via Caetani. However, the above-mentioned reconstruction of Moro's death is still controversial and highly debated (Grassi, 2018; Moro CA, 1998; Provvisionato and Imposimato, 2008; Fasanella and Rocca, 2003). Here we ask: is it scientifically feasible to provide at least a few answers about Moro's death, from a forensic perspective? A recently described, novel topological tool comes into play: proximal planar vortex 1-cycles, which resemble the structure of vortex atoms introduced by William Thomson (Lord Kelvin) in 1867 and recent work on the proximity of shapes that overlap either spatially or descriptively (Peters, 2016). We show, based on novel analysis carried on the photographs taken during the procedure, that the brigatists' account cannot hold true, because Moro was killed in three steps. 


\section{MATERIALS AND METHODS}

The recently-introduced computational topology technique. A vortex cycle is acollection of non-concentric, nesting, possible overlapping cyclic skeletons (path-connected vertexes arranged in a sequence of line segments, where a path occurs between each pair of skeleton vertexes).Overlapping cyclic skeletons yield anAlexandroff nerve (Alexandroff 1965; Alexandroff and Hopf, 1935).Overlapping vortex cycles constitute a vortex nerve complex. This is a very basic form of vortex nerve (Peters 2018a; Peters 2018b). By considering converging rays of light reflected from surface shapes, we arrive at what is known as an optical vortex nerve (Peters 2019), useful in detecting, analyzing and classifying surface shapes (Ahmad and Peters, 1917; Peters 2017) such as those commonly found in forensic images.

In the search for good approximations of surface shapes, it helps to imitate the envelope created by a light caustic that is reflected off a shiny surface (Figure 1A). After all, we want to cover an unknown shape on a curved surface displayed in a digital image with a known geometric shape. The closer a geometric shape covering is to a surface shape, the more effective the geometric shape will be in approximating the surface shape. It has been noticed that a light caustic results from light reflected from a curved surface (Nye 1999; Nye 2018) (Figure 1B). In general, F.J. Wright (1977) observes that a light caustic is the envelope along which geometrical light rays coalesce.

The basic idea in designing an optical vortex nerve is to arrive at a minimalistic geometric shape useful in approximating an unknown surface shape commonly found in forensic images. We start with finding the barycenters of the triangles on a triangulated surface shape. The vertices of the innermost cyclic skeleton in, for example, the optical vortex nerve in Figure 1C, is located on triangle barycenters. The outermost cyclic skeleton in the simplest of optical vortex nerves is located on the barycenters of the triangles shown. Each triangle vertex is the centroid of a hole (a surface region that absorbs light) and the triangle barycenters mark the paths of light reflection from a surface shape, such as the boundary of bullet hole. Each change in the number of centroids (centers of mass of light absorption regions) in a forensic image leads to a modification in the number of cusps and folds in the resulting optical vortex nerve. As a result, a change in the number of centroids leads to a variation in the number of nerve triangles (Figure 1D). For further technical issues and details, see Peters (2019).

Autoptic reports and photographs. When autoptic examinationwas performed, Moro was wearing the same clothes he had during the kidnapping. We examined the photographs taken during the body inspection (Questura di Roma, 1978), performed in the first hours of the afternoon of 9 May 1978, the day of the discovery (Figure 2A). Furthermore, we went thoroughly throughthe official autoptic report(Commissione, 1989). These data are freely available on Gero Grassi's website: http://www.gerograssi.it/cms2/index.php. 
A

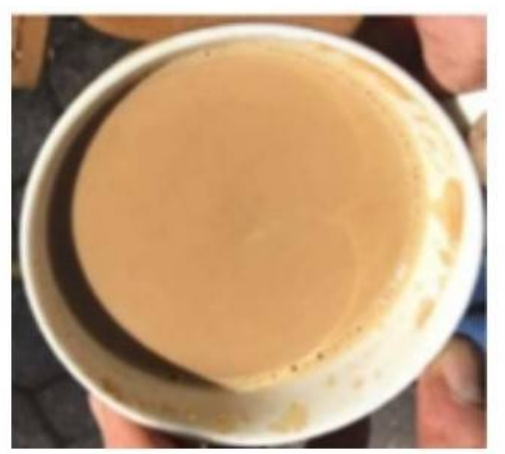

B

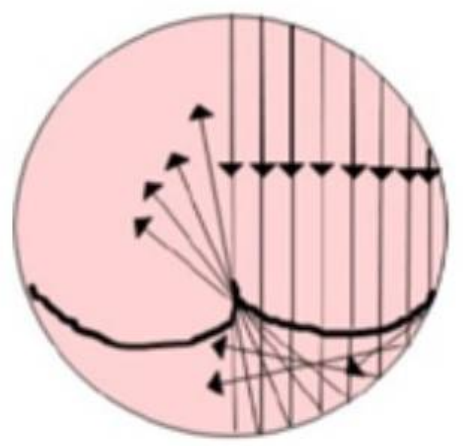

C

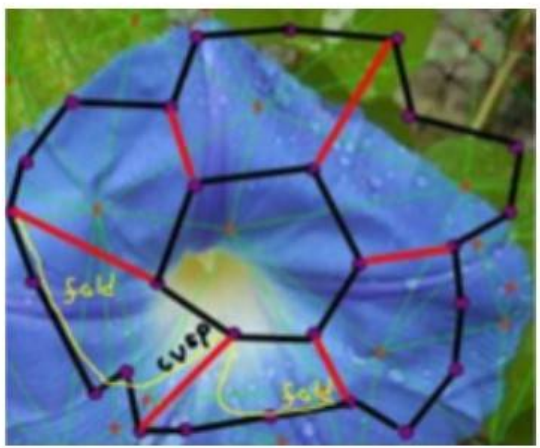

D

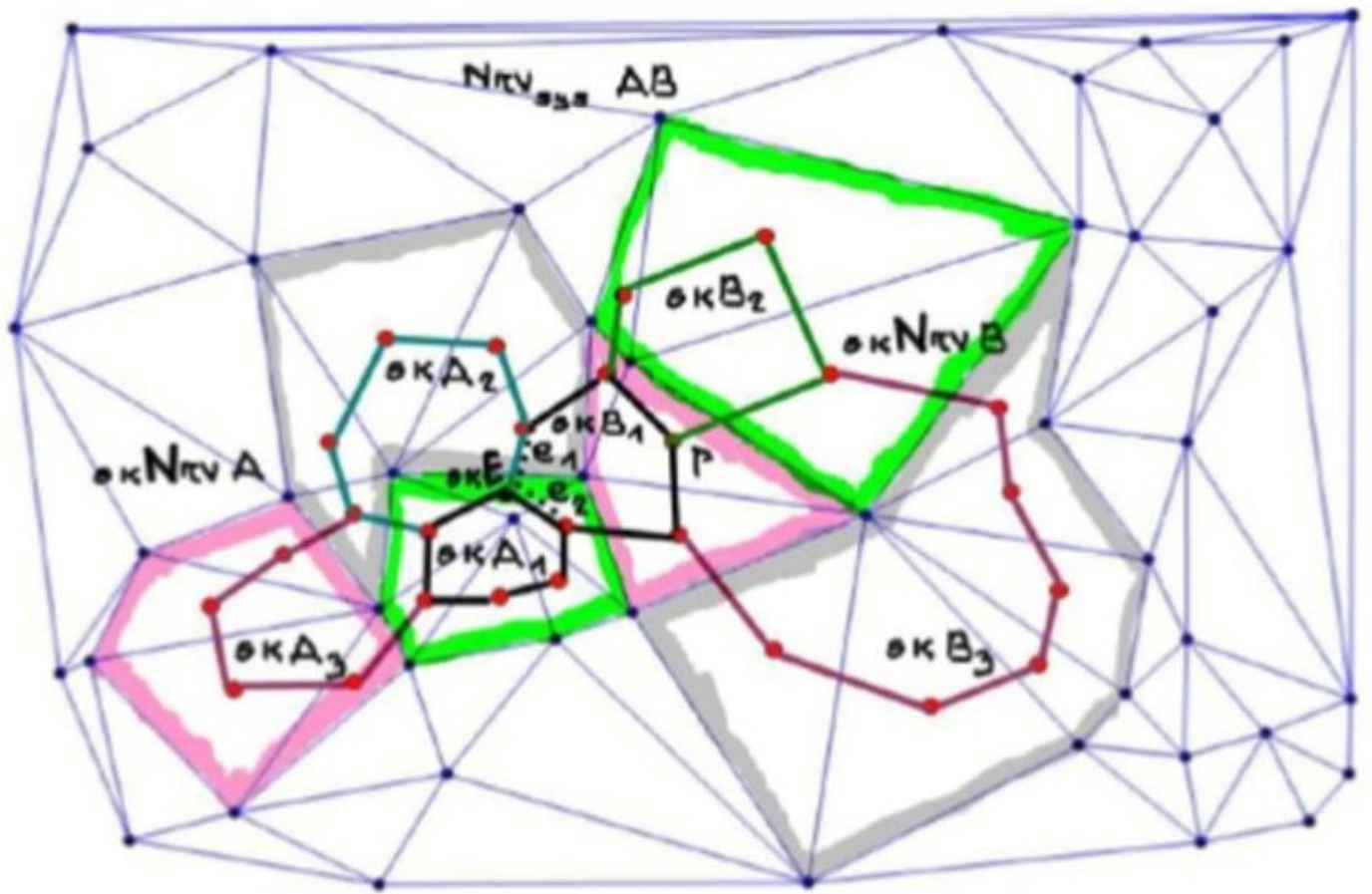

Figure 1. Sample Optical Vortex Nerve. 1A: A light caustic appears here on a coffee surface formed by a collection of converging rays of sunlight reflected off the inner walls of a coffee. 1Billustrates the geometry of the reflected light rays forming a Nye coffee cup caustic. Its tails are called folds and their center is termed a cusp.1C: Optical vortex nerve on a morning glory surface.Light caustic folds and cusp are represented by a pair of path-connected skeletons attached to a central skeleton called the cusp of an optical vortex nerve. Such a nerve is derived from a pair of vortex skeletons with a web of light caustic-like skeletons along its border. A repetition of the filament structures derived from the light caustic is found in the nested, non-concentric cyclic filament skeletons (skeletal vortexes) attached to connecting edges and spokes. It is partially represented by the intersecting yellow lines climbing up one of the spokes shown here. The upper end of each of the spokes represents the cusp of a light caustic that sits on the curved shape of a petal.1D:An example of a system of intersecting skeletal nerves skNrvsys AB. A pair of intersecting skeletal nerves skNrv A and skNrv B are shown. The dotted line indicates line segments that this pair of nerves have in common. 


\section{RESULTS}

Topological investigation on the photographs allowed us to achieve a series of overlapping triangulations displaying closeness and continuity (Figure 2B).

Spatial location of the series of shots. Starting from the optical vortex nerves depicted inFigure 2B, we were able to recognize different series of spatially distinct skin lesions(Figure 1C). In other words, the eleven cutaneous breaks on the President's thorax correspond to three different groups of bullets which were shot together. Based on the abovementioned proximity topological arguments, we identified three series of cutaneous breaks(standing for the shotsthat reached Moro's left thorax) with matching spatial description:

1) $1-2-3-4-7$

2) $9-10-11$

3) $5-8$

Temporal location of the series of shots. Once established the presence of three spatially-separated series of shots, is it possible to assess their temporal sequence? The above-described topological technique does not provide this answer, because temporal sequences cannot be represented by the vortexes. However, the shots'timeline can be inferredthrough the examination of complementary clues, i.e.,the analysis of the autoptic report and other pictures of body and dresses. Going through these documents, we noticed that just two of the eleven bullets pierced the jacket. The two holes on the jacket can be superimposed to just two of the eleven holes penetrating the underlying dresses and Moro's skin: the ones termed 1 and 2. For further details, see Figure 3, which also elucidates why Moro's trunk stood erect during the first series of shots.

During the body inspection, after the removal of the gilet, the cravat looked ruffled and disarrayed (Figure 4A). The explanation is straightforward, if we take into account thatthe doctors reported the presence of napkins under the gilet, assembledin an effort to stop a previous bleeding. Indeed, the cravat's displacementcould be caused by the insertion of the napkins under the gilet after the first shots 1-2-3-4-7 (Figure 4B). Looking at the breaks detectable on the cravat, it can be noticed that they were produced by bullets exploded after the insertion of napkins. These holes correspond to the ones termed 5, 6 and 8 (Figure 5). Therefore, the series 5-8 (including also 6) must have occurred after the 1-2-3-4-7 shots. Concerning the bullets 9-10-11, this serieswas shot when Moro's body was inside the car, because their matching bullets were found stuckin the trunk's metal underlying the corpse.

Therefore, the temporal sequence of the three shots sequences was:

1) $1-2-3-4-7$

2) $5-8$

3) $9-10-11$ 

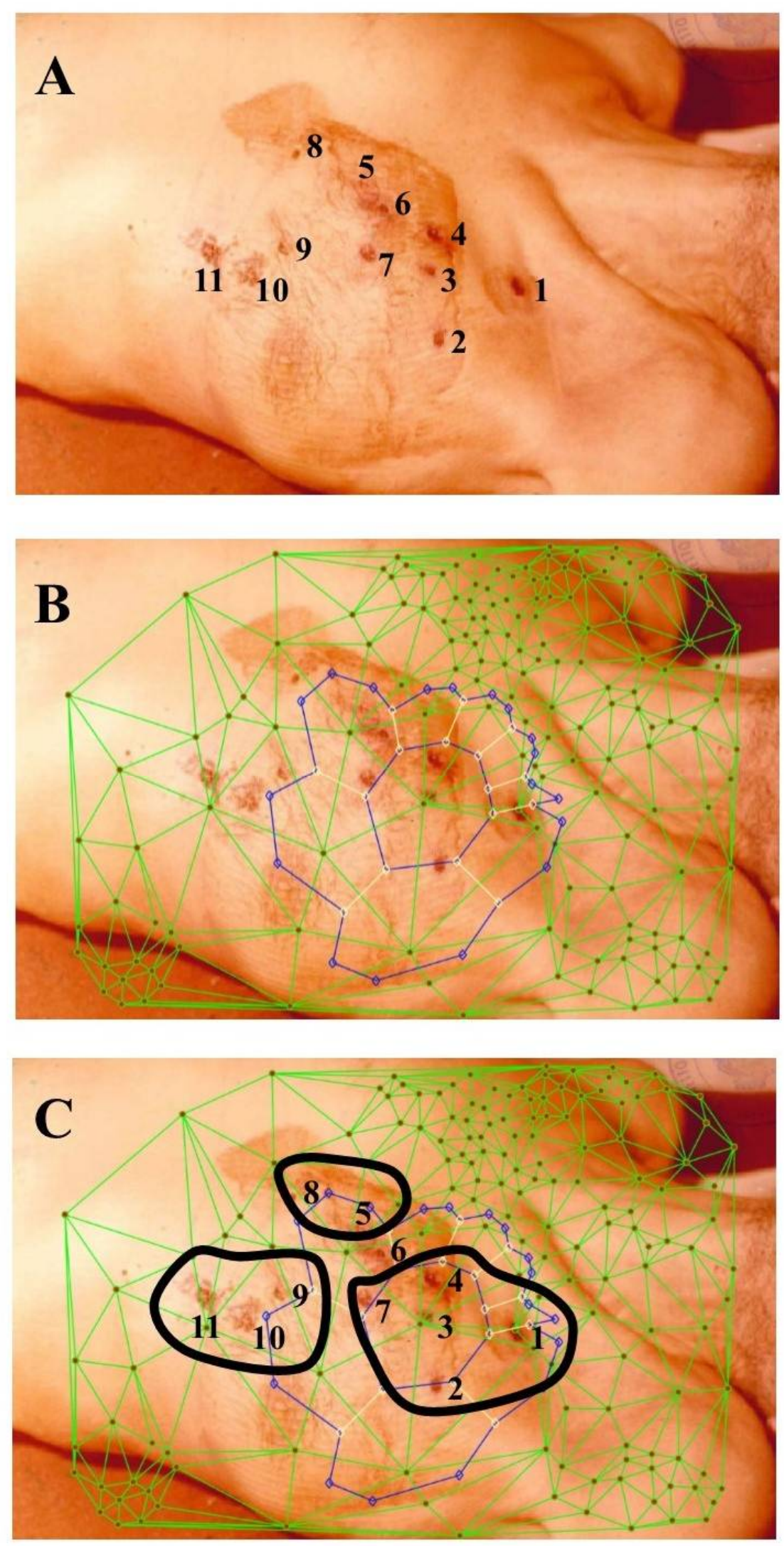

Figure 2A. Cutaneous breaks on the anterior surface of Moro's left anterior thorax. Note thatthe numeration of the holes is slightly different from the one described in the autoptic report, because we preferred to use the standard classification found in books discussing the Moro Affair. 
Figure 2B.Tessellation performed at 84 sites. An optical vortex nerve structure consists of a nucleus surrounded by compartments that represent the paths of light reflected from surfaces recorded by a camera.

Figure 2C.Topological analysis reveals that the cutaneous breaks can be subdivided in three spatial sequences, corresponding to three series of temporally separated shots.
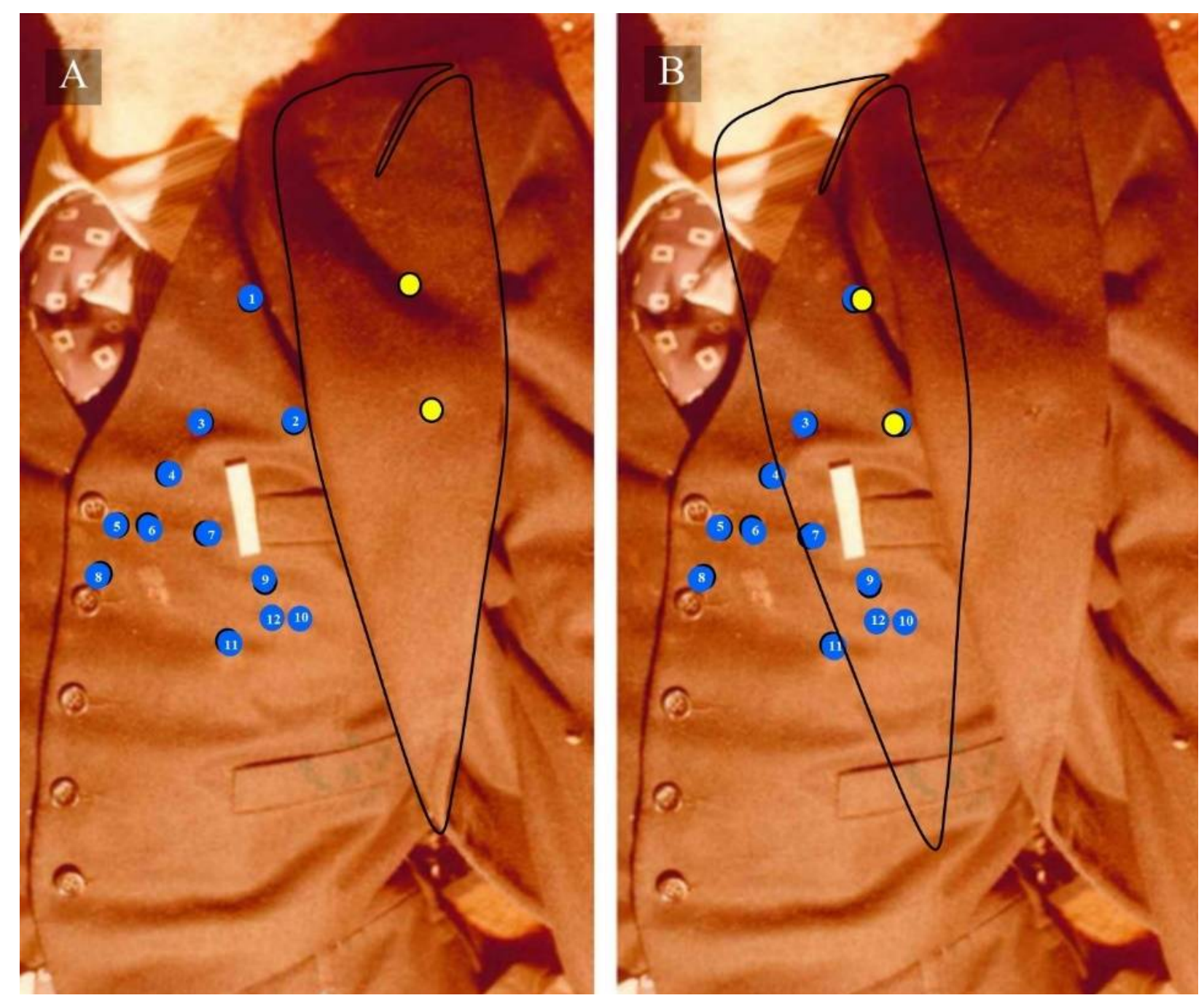

Figure3A. This picture, taken during the forensic body recognition, illustrates the eleven entry breaks on the gilet (blue spots). The numeration of these holes follows the one described in Figure 1,concerning cutaneous lesions. The two holes passing through the jacket are marked in yellow. Note that in this picture, taken after the body retrieval, the jacket does not display the same locationheld by the body during the first shooting. Figure3B. Just two blue holes on the gilet(the ones termed 1 and 2)match the yellow breaks on the jacket. Because the proper jacket's location during shots corresponds to an individual standing vertical, this means that, when Moro was reached by the first bullets, he was seated or upright. 

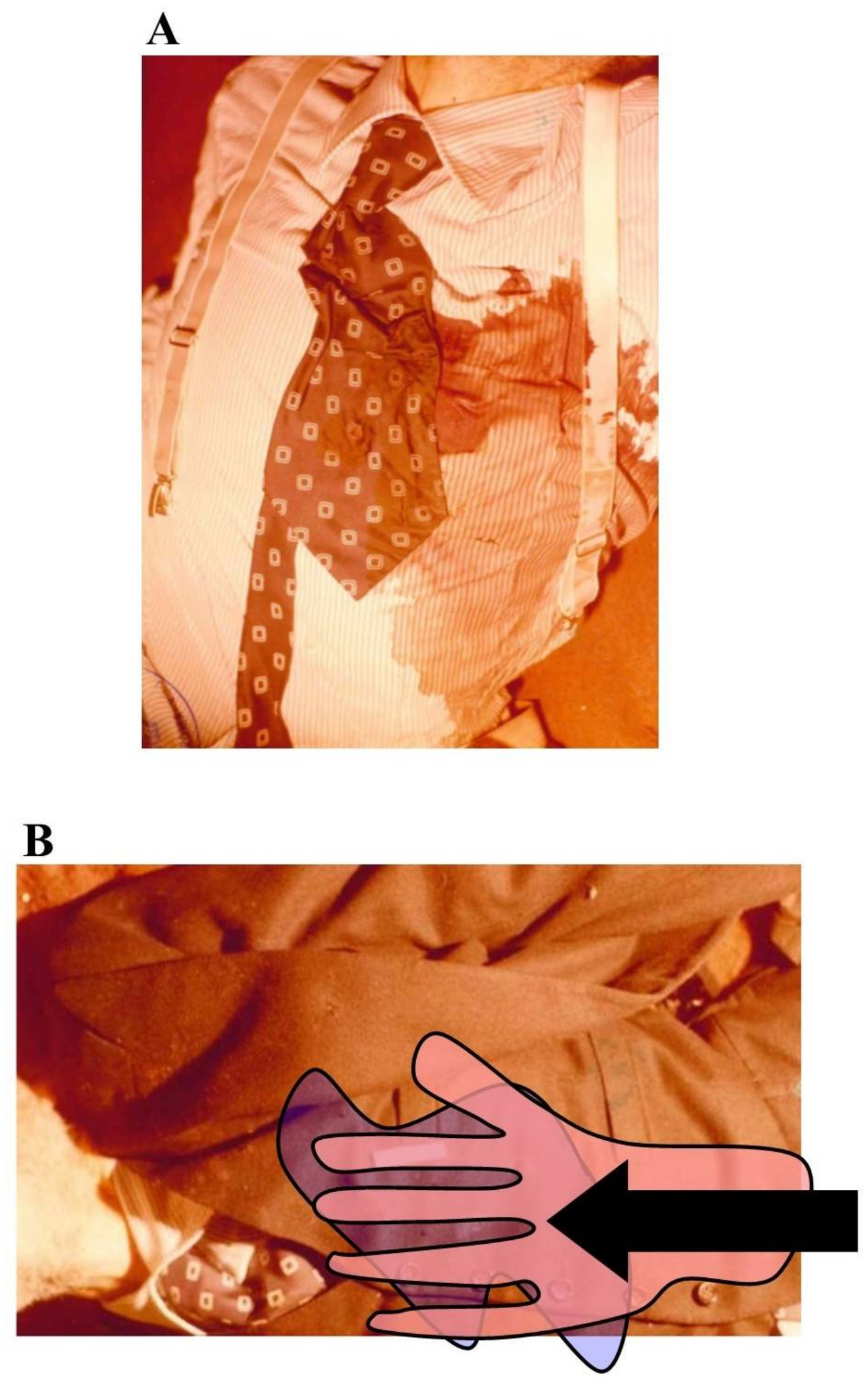

Figure 4. The cravat has been found warpedand dislocated $(\mathbf{4 A})$, due to the napkins inserted under the giletafter the first shots(4B), in order to stop the bleeding from the underlying cutaneous breaks 1-2-3-4-7. 


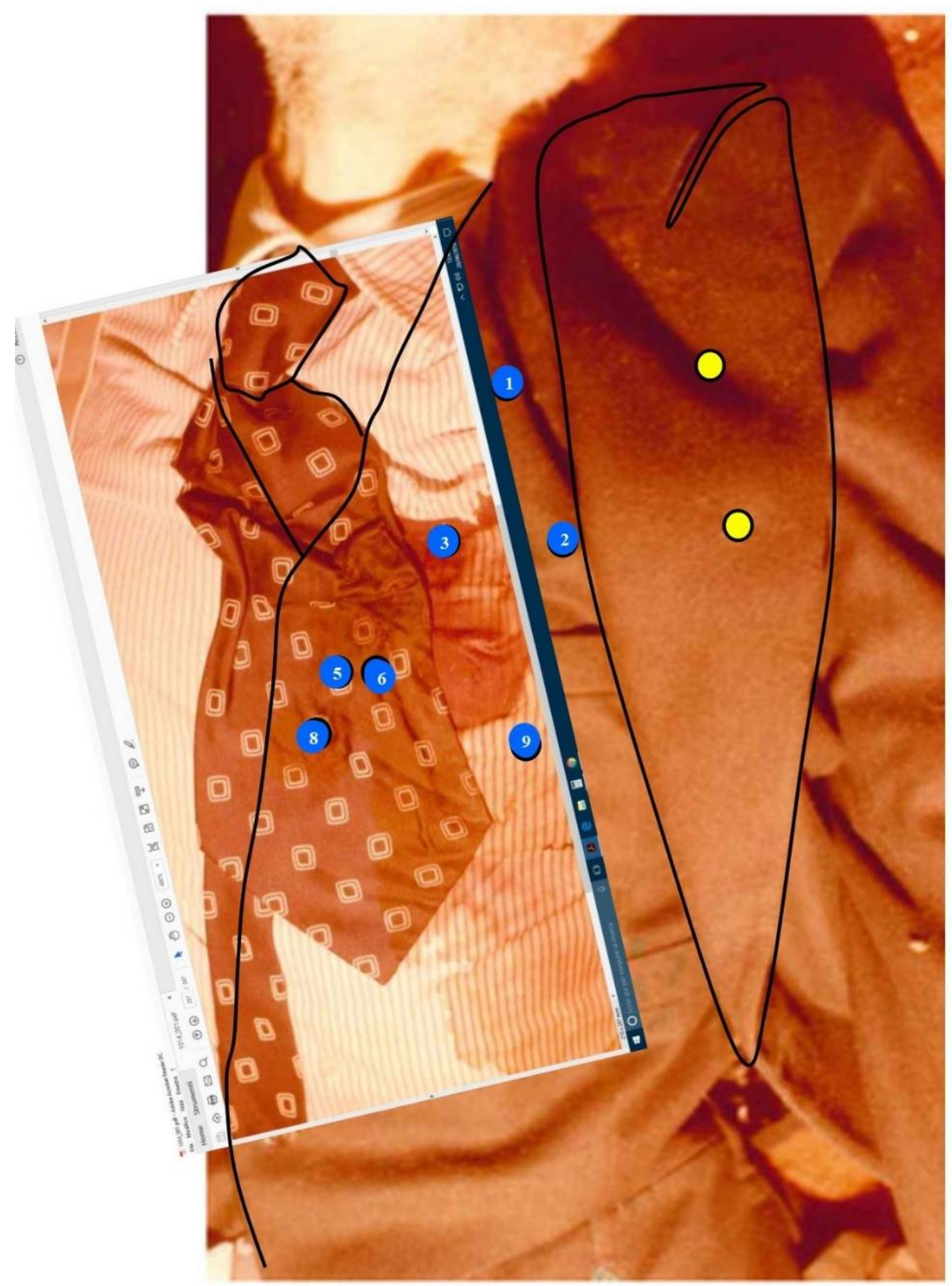

Figure 5. A computerized reconstruction of the cravat in the proper position under the gilet. The borders of the gilet and jacketare highlighted (black lines). Note that the holes on the ruffled cravat match the shoots 5,6 and 8. This means that the latter were exploded after the series 1-2-3-4-7. 


\section{CONCLUSIONS}

Our results, contrary to the official version ofone-step execution occurring inside the car's trunk with Moro's body lying supine, point towards a three-steps execution. According to our analysis, the temporal series of the shots was the following: at first the President, standing upright or seated, was shot by the bullets that reached the cutaneous holes 1-23-4-7. Then the killers, in an effort to stop the hemorrhage, introduced a few napkins under the gilet. It followed the sequence of shots 5-8 (and 6), which pierced the cravat dislocated by the towels. The final sequence, corresponding to the shots 9-10-11, was thrown when the Moro's body was already located supine, inside the car's trunk. Another detail points towards a multi-step execution: when Moro was found in the trunk twisted round theleft side, his RIGHThand was covered by blood. This means that the President, after the first shots, raised the right arm to shelter the left thorax. Further, our work demonstrates that the novel technique is able to detect dominant, light-reflecting surface shapes in the neighborhoods of light-absorbing surface regions such as bullet holes. An optical vortex nerve derived from the reflected light of surface shapes captured in a camera image tends to cover those forensic surface regions of interest. The secret to deriving forensically useful optical vortex nerves is the focus on regions of a triangulated image that have the highest number of triangles with a common vertex (called the nucleus of a barycentric Alexandroff nerve) (Alexandroff 1935). This is the case, since the resulting maximal optical vortex nerves reveal those surface shapes in a forensic image that are sources of the highest number of reflected light rays. The analysis method introduced in this paper provides a means of finding dominant light-reflecting surface shapes, containing the highest concentration of forensic information signatures. What next? We suggest that this topological technique could be used also to solve analogous cases in which forensic pictures are available: to provide a theoretical example, it would be feasible to describe the temporal series of shots that reached Benito Mussolini's corpse during his controversial execution.

\section{AKNOWLEDGMENTS}

The Authors would like to thank the Team SEDICIdiMARZO (http://www.sedicidimarzo.org/) and Gero Grassi for their skillful suggestions and precious insights.

\section{REFERENCES}

1) Ahmad MZ, Peters JF. 2017. Proximal Cech complexes in approximating digital image object shapes. Theory and application, Theory and Application of Math. \& Comp. Sci. 7 (2), 81-123, MR3769444.

2) Alexandroff P. 1965. Elementary Concepts of Topology. Dover Pubs., Inc., NY, translation of EinfachsteGrundbegriffe der Topologie, Springer, 1932, translated by A.E. Farley, Preface by D. Hilbert, MR0149463.

3) AlexandroffP,Hopf H. 1935. Topologie. Band I, Springer, Berlin. Zbl 13, 79, MR0345087.

4) Biscione F. 1993. Il memoriale di Moro rinvenuto in Via Monte Nevoso a Milano. Coletti Editore, Rome.

5) Commissione Parlamentare d'inchiesta sulla strage di via Fani sul sequestro e l'assassinio di Aldo Moro e sul terrorismo in Italia. 1989. Verbale di descrizione ed identificazione del cadavere e di autopsia (Aldo Moro). Vol. 45, Doc. XXIII, n.5: 649-662.

6) Drake R. 1995. The Aldo Moro Murder Case. Cambridge, Massachusetts: Harvard University Press. ISBN 0674-01481-2.

7) Fasanella G, Rocca G. 2003. Il misterioso intermediario. Igor Markevic e il caso Moro. Turin: Einaudi. ISBN 88-06-16087-7.

8) Gotor M. 2018. Aldo Moro- Lettere dalla Prigionia. ed. "Gli Struzzi”, Giulio Einaudi Editore, Torino.

9) Grassi S. 2008. Il caso Moro. Un dizionario italiano. Milan: Mondadori.

10) Grassi G. 2018. Aldo Moro: la verità negata. CSL Pegasus Edizioni

11) Martinelli R, Padellaro A. 1979. Il Delitto Moro. Rizzoli.

12) Montanelli I, Cervi M. 1991. L'Italia degli anni di piombo. Milan, Rizzoli Editore.

13) Moro AC. 1998. Storia di un delitto annunciato. Le ombre del caso Moro. Editori Riuniti, Roma.

14) Nye J. 1999. Natural Focusing and Fine Structure of Light. Caustics and Dislocations. Institute of Physics Pub., Bristol, MR1684422.

15) Nye J. 2018. Events in fields of optical vortices: Rings and reconnection. Journal of Optics 18, 1-11, DOI: 10.1088/2040-8978/18/10/105602. 
16) Peters JF. 2016. Computational proximity. Excursions in the topology of digital images., Intelligent Systems Reference Library 102 (2016), xxviii + 433pp,ISBN: 978-3-319-30260-7; 978-3-319-30262-1; DOI: 10.1007/978-3-319-30262-1; zbMATHZbl1382.68008;MR3727129.

17) Peters JF. 2017. Proximal planar shape signatures. Homology nerves and descriptive proximity, Advan. in Math: Sci. J 6 (2), 71-85, Zbl 06855051.

18) Peters JF. 2018a. Proximal Vortex Cycles and Vortex Nerve Structures. Non-Concentric, Nesting, Possibly Overlapping Homology Cell Complexes. Journal of Mathematical Sciences and Modelling, 1-17, http://dergipark.gov.tr/jmsm [in press].

19) Peters JF. 2018b. Proximal planar shapes. Correspondence between shape and nerve complexes, arXiv 1708 (2017), no. 04147v1, 1-12, Bulletin of the Allahabad Math. Soc., Dharma Prokash Gupta Memorial Volume, 33(1), 2018, 113-137.

20) Peters JF. 2019. Computational Geometry and Topology of Digital Images. Shape Complexes, Optical Vortex Nerves and Proximities. Intelligent System Reference Library, Springer Int. Publishing AG [in press].

21) Provvisionato S, Imposimato F. 2008. Doveva morire. Chi ha ucciso Aldo Moro. Il giudice dell'inchiesta racconta. Chiarelettere. ISBN 978-88-6190-025-7.

22) Questura di Roma. 1978. Fascicolo dei rilievi tecnici eseguiti in giorno 9 Maggio 1978, in via Michelangelo Caetani, ove è stata rinvenuta l'autovettura Renault con a bordo il cadavere dell'On. Aldo Moro. Gabinetto Regionale di Polizia Scientifica.

23) Satta V. 2006. Il caso Moro e i suoi falsi misteri. Rubbettino, Soveria Mannelli.

24) Sciascia L. 1994. L'affaire Moro. Adelphi.

25) Wright F. 1977. Wavefield singularities: a caustic tale of dislocation and catastrophe. Ph.D. Thesis, University of Bristol, H.H. Wills Physics Laboratory, Bristol, England, https://researchinformation.bristol.uk/files/34507461/56229.pdf.

26) Zavoli S. 1992. La notte della Repubblica. Rome, Nuova Eri. 